\title{
Internal strains and crystal structure of the layers in AIGaN/GaN heterostructures grown on a sapphire substrate
}

V. P. Kladko, A. F. Kolomys, M. V. Slobodian, V. V. Strelchuk, V. G. Raycheva, A. E. Belyaev, S. S. Bukalov, H. Hardtdegen, V. A. Sydoruk, N. Klein, and S. A. Vitusevich

Citation: Journal of Applied Physics 105, 063515 (2009);

View online: https://doi.org/10.1063/1.3094022

View Table of Contents: http://aip.scitation.org/toc/jap/105/6

Published by the American Institute of Physics

\section{Articles you may be interested in}

Crystalline structure of AIGaN epitaxy on sapphire using AIN buffer layers

Applied Physics Letters 65, 2302 (1994); 10.1063/1.112724

Mechanism of strain relaxation by twisted nanocolumns revealed in AIGaN/GaN heterostructures

Applied Physics Letters 95, 031907 (2009); 10.1063/1.3184569

Strain relaxation in AIGaN under tensile plane stress

Journal of Applied Physics 88, 7029 (2000); 10.1063/1.1326852

Elastic strain relaxation and piezoeffect in GaN-AIN, GaN-AIGaN and GaN-InGaN superlattices Journal of Applied Physics 81, 6332 (1997); 10.1063/1.364368

Two-dimensional electron gases induced by spontaneous and piezoelectric polarization charges in N- and Gaface $\mathrm{AlGaN} / \mathrm{GaN}$ heterostructures

Journal of Applied Physics 85, 3222 (1999); 10.1063/1.369664

Elastic properties of zinc-blende and wurtzite AIN, GaN, and InN

Journal of Applied Physics 82, 2833 (1997); 10.1063/1.366114

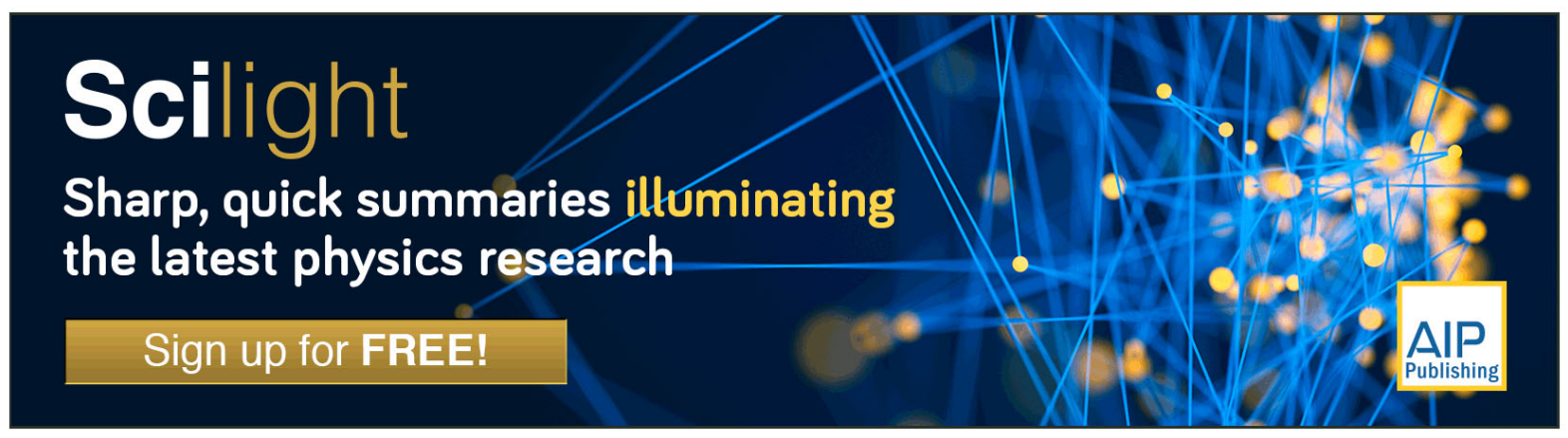




\title{
Internal strains and crystal structure of the layers in AIGaN/GaN heterostructures grown on a sapphire substrate
}

\author{
V. P. Kladko, ${ }^{1}$ A. F. Kolomys, ${ }^{1}$ M. V. Slobodian, ${ }^{1}$ V. V. Strelchuk, ${ }^{1}$ V. G. Raycheva ${ }^{1}$ \\ A. E. Belyaev, ${ }^{1}$ S. S. Bukalov, ${ }^{2}$ H. Hardtdegen, ${ }^{3}$ V. A. Sydoruk, ${ }^{3}$ N. Klein, ${ }^{3}$ and \\ S. A. Vitusevich ${ }^{3, a)}$ \\ ${ }^{1}$ V. Lashkaryov Institute of Semiconductor Physics, NAS of Ukraine, Kiev 0328, Ukraine \\ ${ }^{2}$ A.N. Nesmeyanov Institute of Organoelement Compounds, Scientific and Technical Center on Raman \\ Spectroscopy, Russian Academy of Sciences, Vavilova str. 28, Moscow 119991, Russia \\ ${ }^{3}$ Institut für Bio- und Nanosysteme and CNI-Center of Nanoelectronic Systems for Information Technology, \\ Forschungszentrum Jülich, D-52425 Jülich, Germany
}

(Received 14 November 2008; accepted 29 January 2009; published online 20 March 2009)

In this paper, we investigate the structural properties of $\mathrm{AlGaN} / \mathrm{GaN}$ heterostructures grown by metal organic chemical vapor deposition on sapphire substrates with different thicknesses using high-resolution x-ray diffraction and Raman scattering methods. We discuss the microscopic nature of spatial-inhomogeneous deformations and dislocation density in the structures. Microdeformations within mosaic blocks and the sizes of regions of coherent diffraction are determined. We reveal a gradient depth distribution of deformations in the mosaic structure of nitride layers, as well as at the interface regions of the sapphire substrate on the microscale level using confocal micro-Raman spectroscopy. We determine that an increase in substrate thickness leads to a reduction in dislocation density in the layers and an increase in the elastic deformations. The features of the block structure of nitrides layers are shown to have a significant influence on their elastic properties. (C) 2009 American Institute of Physics. [DOI: 10.1063/1.3094022]

\section{INTRODUCTION}

Epitaxial III-nitride heterostructures are the most promising material system for high-temperature high-power electronic and optoelectronic applications. ${ }^{1}$ Despite recent progress in the growth optimization of III-nitride layers on sapphire substrates, the large lattice mismatch and the difference in thermal expansion coefficients between substrate and films led to the formation of layers with a large concentration of structural defects (with dislocation densities of the order of $\approx 10^{8}-10^{10} \mathrm{~cm}^{-2}$ ) as well as significant residual deformations. ${ }^{2,3}$ It has recently been shown that III-nitride layers grown on an $\mathrm{Al}_{2} \mathrm{O}_{3}$ (0001) substrate have a predominantly mosaic structure consisting of $c$-oriented nanocolumns. ${ }^{4,5}$ Furthermore the size dependence of Young's modulus has been revealed in nanocolumn structures, such as $\mathrm{ZnO}$ nanowires with a diameter of $<120 \mathrm{~nm}$ (Ref. 6) or GaN nanotubes. ${ }^{7}$ This effect is caused by an increased hardness of the nanocolumn surface due to the decrease in the bond length near the surface. ${ }^{8}$ It is known that peculiarities in the mosaic structure of nitride layers determine their electrical properties. ${ }^{9}$ However, up to now, no established physical mechanism could be pinpointed to explain the features in the mosaic structure of III-nitride heterostructures, which has considerably postponed progress in this field of research. In order to solve this problem, a detailed study of the influence of the deformation state must be performed on the structural and electrical properties of multilayered nitride heterostructures and their mosaic structure and on defects caused by

\footnotetext{
${ }^{a)}$ Electronic mail: s.vitusevich@fz-juelich.de.On leave from the Institute of Semiconductor Physics, NASU, 03028 Kiev, Ukraine.
}

elastic strain relaxation processes. These studies are necessary to qualitatively improve the properties and parameters of devices based on III-nitride heterostructures.

However, traditional methods used for structural investigations do not provide detailed enough information on a mosaic structure. X-ray diffraction (XRD) is the most effective method of obtaining structural parameters averaged over the volume, magnitude of elastic strain deformation, and composition of the nitride structures. ${ }^{10}$ An analysis of the XRD rocking curves in different geometries allows us to characterize the orientation distribution of the structural defects. ${ }^{11}$ For the investigation of mosaic structures, it is particularly important to investigate the local three-dimensional (3D) distribution of a crystalline phase, the local texture and the deformation tensor with a spatial resolution below $1 \mu \mathrm{m}$. The method of 3D XRD microscopy allows crystalline materials to be investigated with micrometer resolution, ${ }^{12}$ and X-ray microdiffraction at high-power synchrotron excitation permits submicron resolution measurements of the deformation tensor in three directions. ${ }^{13}$

Raman scattering spectroscopy is a nondestructive tool for the qualitative and quantitative analysis of residual deformations in nitride epilayers. There are no publications on this subject for mosaic structures of the nitrides. Elastic deformations in strained materials cause changes in the binding constants and a change in the frequencies of the optical phonons, respectively. Under strain, the shift of the optical phonon frequency from its position in unstrained material is registered in the Raman spectrum. This Raman shift allows the internal strain sign and magnitude to be estimated. For wurtzite III-nitride structures, the frequency of the nonpolar $E_{2}^{\text {high }}$ mode is strongly affected by residual deformations in- 
duced in the epilayers of $\mathrm{GaN},{ }^{14,2} \mathrm{AlN},{ }^{15}$ and $\mathrm{AlGaN}$ (Ref. 16) mainly due to the different thermal expansion coefficients of the epilayer and the substrate, as well as their lattice mismatch. Modern laser Raman spectrometers are equipped with ultrasensitive charge-coupled device (CCD) detectors and microscopes, which allow the analysis of the spatial nonuniformity and strain of the structure (for example, silicon or other semiconductors) and heterostructures with a resolution down to a few microns. Moreover, it was recently demonstrated for transparent materials that micro-Raman spectroscopy using a confocal microscope configuration with submicrometer positioning of the excitation laser beam allows the 3D distribution of strains to be investigated with a lateral resolution of 100-500 $\mathrm{nm}$ and the scan to be controlled step by step to a depth of a few microns with an axial resolution of 100-600 nm. ${ }^{17}$ The efficiency of confocal micro-Raman spectroscopy was recently demonstrated in an investigation of the depth distribution of deformations in plastically deformed sapphire crystals with a $300 \mathrm{~nm}$ lateral resolution and an axial depth resolution of around $600 \mathrm{~nm}^{18}$

Despite the significant interest in device applications based on III-nitride heterostructures, it is still unclear as to how structurally perfect epitaxial layers can be obtained. In order to find a solution to this problem, different complementary methods must be used to perform a comprehensive study.

In this paper, we investigate the influence of the sapphire substrate thickness on the structural parameters and on the deformation state in individual layers in $\mathrm{Al}_{x} \mathrm{Ga}_{1-x} \mathrm{~N} / \mathrm{GaN}$ heterostructures using XRD analysis and micro-Raman spectroscopy. The results obtained help to explain the significant differences in deformation constants for III-nitride heterostructures, which have been experimentally determined by different authors and can be found in literature.

\section{EXPERIMENTAL METHODS}

The epitaxial $\mathrm{AlGaN} / \mathrm{GaN}$ heterostructures under investigation were grown by metal organic chemical vapor deposition on (0001) sapphire $\left(\mathrm{Al}_{2} \mathrm{O}_{3}\right)$ thick substrates (TKs) and thin substrates (TNs) with thicknesses of 3 and $0.45 \mathrm{~mm}$, respectively. After the deposition of a $3-\mu \mathrm{m}$-thick $\mathrm{GaN}$ buffer layer, an $\mathrm{Al}_{0.30} \mathrm{Ga}_{0.70} \mathrm{~N}$ layer with a thickness of $30 \mathrm{~nm}$ was grown, followed by $4 \mathrm{~nm} \mathrm{GaN}$ cap layer. The misorientation of the substrate surface did not exceed 30 angular minutes.

The structural quality of the samples was investigated by high-resolution triple-axis diffractometry. ${ }^{19}$ The experimental setup allowed obtaining the two cross sections of reciprocal lattice nodes-orthogonal to the diffraction vector ( $\omega$-scan) and parallel to it $(\omega / 2 \theta$-scan). The measurements were performed on "PANalytical X'Pert PRO MRD" equipment using symmetric 0002, 0004 and asymmetric 10-12 and $-1-124$ reflections.

The asymmetric Bragg geometry allowed the properties of symmetric Bragg diffractions to be combined with Laue diffractions. The effects of interplanar spacing changes and atomic plane turns can be studied separately using triple-axis diffractometry. Therefore, an analysis of the intensity distri- bution in the $\left(q_{Y}, q_{X}\right)$ coordinate system, directed along and orthogonal to the $\boldsymbol{H}$ vector, respectively, allows us to distinguish the contribution made by each of the effects. ${ }^{20}$ The surface morphology of the heterostructures was investigated using atomic force microscopy (AFM).

The macrodeformations induced by bending the sample are important characteristics of the GaN layer. Since the layer thickness is much smaller than the substrate thickness, the curvature radius of the layer is assumed to be like that of the substrate. The latter can be determined by measuring the deviation of the angle of a sapphire reflection at a linear scanning of the sample under a constant x-ray beam.

The micro-Raman measurements were carried out in backscattering geometry at room temperature with the Raman spectrometer Jobin Yvon LabRam, equipped with an Olympus confocal optical microscope, a TV camera, and a TE-cooled CCD for detection. The excitation light source was a $\mathrm{He}-\mathrm{Ne}$ laser of $632.8 \mathrm{~nm}$ wavelength and the beam was focused onto the sample with an optical microscope [100 magnification, numerical aperture $(\mathrm{NA})=0.9]$, which allowed us to focus the laser beam waist to about $1 \mu \mathrm{m}$ in diameter. The maximum laser power on the sample did not exceed $1 \mathrm{~mW}$. Raman peak positions were determined with an error of less than $0.15 \mathrm{~cm}^{-1}$.

\section{EXPERIMENTAL RESULTS}

Two-dimensional (2D) maps of the intensity distribution around the nodes of a reciprocal lattice obtained in three-axis configuration for two AlGaN/GaN heterostructures are shown in Fig. 1. On these maps of intensity measured for (0002) [Figs. 1(a) and 1(b) and (-1-124) [Figs. 1(c) and 1(d)] reflections, two diffraction maxima are seen corresponding to GaN and AlGaN films. Additionally, the interference pattern of the intensity redistribution along vector $\boldsymbol{H}$ can be resolved for the symmetrical reflection 0004. For the $(-1-124)$ reflection, the interference pattern splits into two separate systems of contours (nearly the nodes of film and substrate). As can be seen in the figure, the intensity redistribution extends along the direction orthogonal to vector $\boldsymbol{H}$ for both systems of contours.

Such intensity redistribution is typical for structures containing nonrelaxed elastic strain and misfit dislocations. ${ }^{21}$ The influence of a dislocation network on Bragg diffraction results is the broadening of the diffraction pattern along the direction orthogonal to the diffraction vector $\boldsymbol{H}$. This effect was resolved for both types of investigated structures. However, the dislocation networks do not change the intensity distribution along the diffraction vector. As shown in Fig. 1, the intensity contours do not only extend along the direction orthogonal to vector $\boldsymbol{H}$, but they also shift along the $q_{Y}$ axis. The latter results in the broadening of the diffraction pattern in the direction orthogonal to vector $\boldsymbol{H}$. The peaks of the layers shift with respect to the substrate peak (not shown in Fig. 1). This is because of a macrodisorientation between the film and the substrate - their reflection planes are not parallel to each other (disorientation is $\approx 0.3^{\circ}$ ). These macrodisorientations are almost identical for both structures under investigation. 

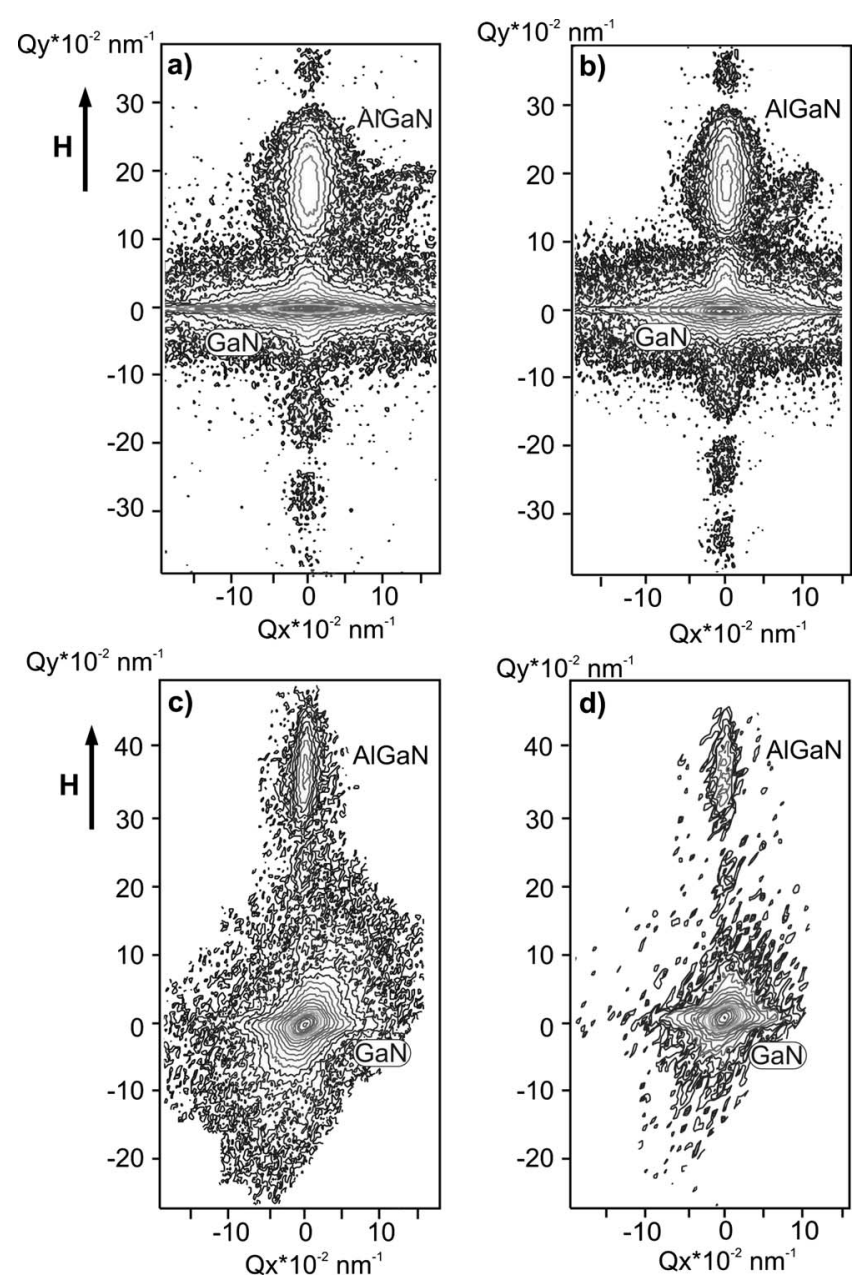

FIG. 1. Intensity distribution around XRD reflections [(a) and (b)] (0002) reflections and [(c) and (d)] (-1-124) reflections for heterostructures grown on [(a) and (c)] thin substrates and [(b) and (d)] thick substrates.

We used the Williamson-Hall method ${ }^{22}$ to determine microdeformations inside the blocks of mosaic structure or nanopillars and the sizes of these blocks, i.e., regions of coherent scattering (RCS). The broadening of symmetrical 0002 and $0004 \omega$-scans was influenced by an inclination and a magnitude of lateral correlation length $\left(L_{\mathrm{II}}\right)$. These two contributions can be distinguished by Williamson-Hall dependence plots. ${ }^{22,23}$ On the other hand, the broadening of $\omega-2 \theta$ scans for these reflections is caused by vertical correlation length $(L)$ and nonuniform strains along the growth direction $\left(\varepsilon_{\perp}\right)$. The linear dependence of Bragg peak broadening on the size of linear blocks and deformations is used to analyze the deformation parameters of the structure for two directions with respect to the diffraction vector $\boldsymbol{H}$,

$$
\beta=1 / D+2 \varepsilon H,
$$

where $\beta$ is the broadening of the reflection peak, $D$ is the size of RCS, $\varepsilon$ is the deformation, and $H$ is the diffraction vector.

Linear dependencies of a full width at half maximum (FWHM) of the reflection curves were plotted as a function of diffraction vector on the basis of Eq. (1). The slopes of these dependencies are related to the contribution of misorientations and deformations. The intersections of these lines with the ordinate axis give the sizes of RCS for two crossorthogonal directions. From the 2D maps of the intensity distribution, the lateral correlation parameter of RCS redistribution was determined. ${ }^{24}$ This parameter was higher for GaN layers grown on TNs than for those grown on TKs. At the same time, this dependence is the opposite for $\mathrm{AlGaN}$ layers: the lateral correlation parameter is higher for the samples grown on thick substrates (see Table I).

The RCS size (height of blocks) in the growth direction for GaN layers grown on TKs is twice as large as that of the layers grown on TNs. The vertical dimensions of RCS for AlGaN layers are practically the same and their height is of the value of nominal thickness. For GaN layers, the values of the deformation parameters and RCS size correlate with the density of edge dislocations. The density of screw dislocations with Burgers vector close to the orthogonal direction with respect to the growth plane are practically the same for GaN layers of these two heterostructures. The components $\varepsilon_{z z}$ of deformation tensor for GaN layers grown on TNs are three times larger than those grown on TKs (Table I). The same relation holds for $\mathrm{AlGaN}$ layers.

It should be noted that the broadening caused by bending can easily be resolved by changing the region's lateral size studied on the sample surface. The contours of equal intensity distribution along vector $\boldsymbol{H}$ reveal several maxima in the region of film reflection [Fig. 2(a)]. These maxima appear due to a change in the interplane distance in the layers of the epitaxial structure or $\varepsilon_{z z}$ tensor components. This demonstrates nonuniformity in the depth distribution of deformation. At the same time, the complex diffraction pattern may appear not only as a result of the regions with different interplane distances but also as a result of the interference of waves scattered at different depths.

Peculiarities in the local distribution of deformations in the growth direction of the structure were investigated using confocal Raman spectroscopy. For a laser excitation wavelength, $\lambda_{\mathrm{exc}}=632.8 \mathrm{~nm}$, the NA of the objective defines the confocal lateral resolution (beam spot diameter) of the Rayleigh diffraction-limited beam and its waist, $\mathrm{d}_{\text {lateral }}$

TABLE I. Structural parameters obtained from the XRD data.

\begin{tabular}{|c|c|c|c|c|c|c|c|c|}
\hline $\begin{array}{l}\text { Parameters/ } \\
\text { sample layers }\end{array}$ & $\varepsilon_{x x}$ & $\varepsilon_{z z}$ & $\varepsilon_{z z} / \varepsilon_{x x}$ & $\begin{array}{c}\text { Correlation } \\
\text { length } \\
(\mathrm{nm})\end{array}$ & $\begin{array}{c}\text { RCS } \\
\text { dimensions } \\
(\mathrm{nm}) \perp / \|\end{array}$ & $\begin{array}{c}\text { Declination angle } \\
\text { of layers plane } \\
\text { (deg) }\end{array}$ & $\begin{array}{l}\text { FWHM } 0004 \\
(\operatorname{deg}) \omega / 2 \vartheta-\omega\end{array}$ & $\begin{array}{l}\text { Dislocation density } \\
\left(\times 10^{-8} \mathrm{~cm}^{-2}\right) \mathrm{E} / \mathrm{S}\end{array}$ \\
\hline GaN TNs & $2.34 \times 10^{-3}$ & $9.04 \times 10^{-4}$ & 0.39 & 52.2 & $31.7 / 58.8$ & 0.38133 & $0.0615 / 0.051$ & $13.8 / 0.98$ \\
\hline AlGaN TNs & $1.52 \times 10^{-3}$ & $6.90 \times 10^{-4}$ & 0.45 & 34.9 & $21.7 / 29.9$ & & $0.107 / 0.1518$ & $2.54 / 2.9$ \\
\hline GaN TKs & $6.75 \times 10^{-4}$ & $3.04 \times 10^{-4}$ & 0.45 & 43.6 & $64.5 / 50.0$ & 0.38108 & $0.0664 / 0.0545$ & $6.2 / 1.09$ \\
\hline $\mathrm{AlGaN}$ TKs & $6.27 \times 10^{-4}$ & $3.79 \times 10^{-4}$ & 0.60 & 36.5 & $20 / 38.3$ & & $0.0815 / 0.1419$ & $2.8 / 1.74$ \\
\hline
\end{tabular}



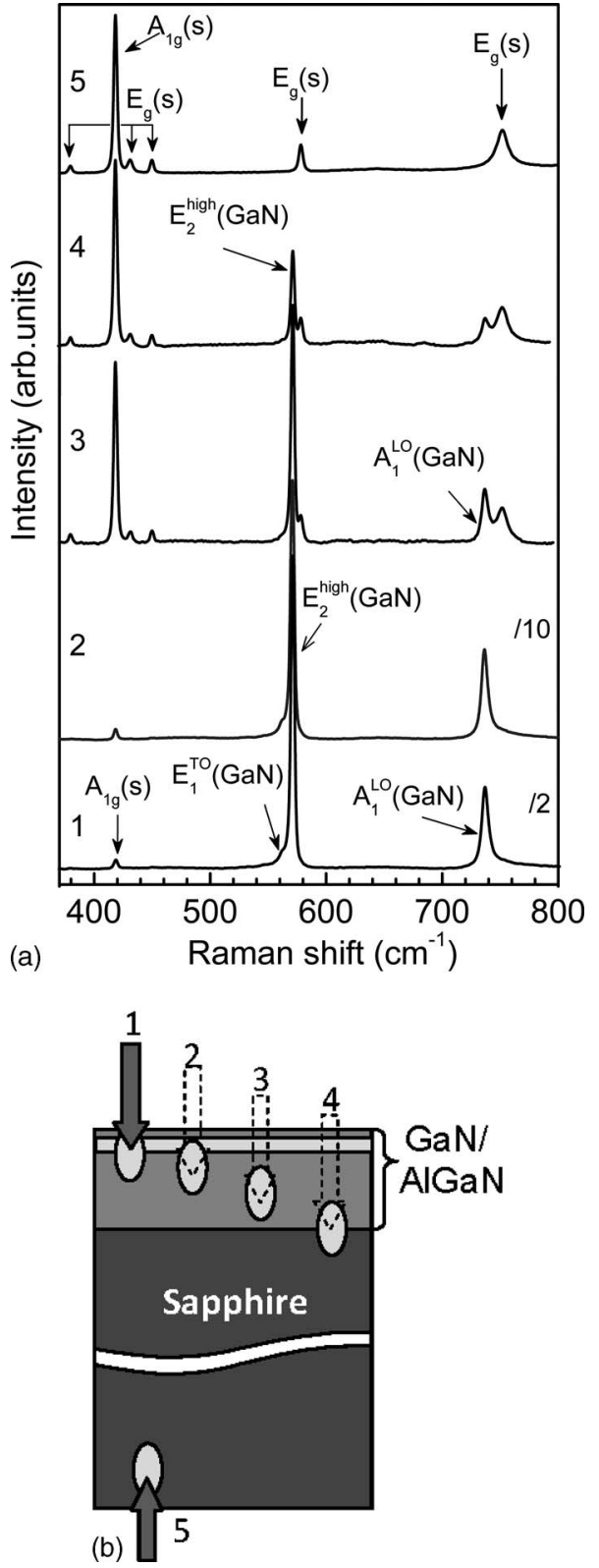

FIG. 2. (a) Raman spectra for different depth scans [points 1-5 in (b)] of an $\mathrm{Al}_{0.30} \mathrm{Ga}_{0.70} \mathrm{~N} / \mathrm{GaN}$ structure grown on a thick sapphire substrate $(3 \mathrm{~mm})$ (curves 1-4). Curve 5-Raman spectrum measured from the back side of this structure: $T=300 \mathrm{~K}$ and $\lambda_{\text {exc }}=632.8 \mathrm{~nm}$. (b) Measurement configuration using the axial focal spot of the laser beam from the front (points 1-4) and back side (point 5) of the investigated structure.

$=1.22\left(\lambda_{\text {exc }} / 2 \mathrm{NA}\right) \approx 430 \mathrm{~nm} .{ }^{17}$ The Raman study provides us with information about the layers of heterostructures with confocal axial resolution $R_{\text {axial }}=1.4\left(\lambda_{\text {exc }} \eta /(\mathrm{NA})^{2}\right)$ $\approx 2.5 \mu \mathrm{m},{ }^{17}$ where $\eta=2.25$ is the index of refraction of $\mathrm{GaN}^{25}$ These depths are in the order of the total thickness of nitride layers for the $\mathrm{AlGaN} / \mathrm{GaN}$ heterostructures under investigation.

The hexagonal wurtzite III-nitride structures grown on sapphire substrates belong to the $C_{6 v}^{4}\left(\mathrm{P}_{3} m c\right)$ space group with four atoms in the primitive unit cell where all atoms occupy $C_{3 v}$ sites. According to the group-theory analysis at the $\Gamma$ point of the Brillouin zone $(\vec{q}=0)$, zone center optical phonons belong to the following irreducible representations: ${ }^{26}$

$$
A_{1}+E_{1}+2 E_{2}+2 B_{1}
$$

Among them, $A_{1}$ and $E_{1}$ modes are both Raman and infrared (IR) active, while the two $E_{2}$ modes ( $E_{2}^{\text {low }}$ and $\left.E_{2}^{\text {high }}\right)$ are only Raman active, and the two $B_{1}$ modes are neither Raman nor IR active (silent modes). Here, the polar $A_{1}$ and $E_{1}$ modes are split into longitudinal optical (LO) and transverse optical (TO) phonons by the macroscopic electric field. Thus, six optical modes, i.e., $A_{1}(\mathrm{LO}), A_{1}(\mathrm{TO}), E_{1}(\mathrm{LO})$, $E_{1}(\mathrm{TO}), E_{2}^{\text {high }}$, and $E_{2}^{\text {low }}$, can be observed for the first-order Raman scattering. Raman scattering spectra were measured at room temperature in $z(x, x) \bar{z}$ backscattering geometry with $z$ direction oriented parallel to the $c$-axis (layers growth direction). This allows the detection of the wurtzite $\mathrm{Ga}(\mathrm{Al}) \mathrm{N}$ $E_{2}$ and $A_{1}(\mathrm{LO})$ modes according to the selection rules. ${ }^{26}$

Figure 2(a) shows the confocal Raman spectra of the $\mathrm{GaN} / \mathrm{Al}_{0.30} \mathrm{Ga}_{0.70} \mathrm{~N} / \mathrm{GaN}$ structure grown on the sapphire TKs. Raman spectra were recorded at different depths (different position of the focal spot), as shown schematically in Fig. 2(b). As the focal spot has position 1-nearly the sample surface-the Raman spectrum (curve 1) shows the two expected phonon lines $E_{2}^{\text {high }}(G a N) \quad\left(\approx 571.4 \mathrm{~cm}^{-1}\right.$ and $\Gamma$ $\left.\approx 3.5 \mathrm{~cm}^{-1}\right)$ and $A_{1}^{\mathrm{LO}}(\mathrm{GaN}) \quad\left(\approx 737.1 \mathrm{~cm}^{-1}\right.$ and $\Gamma$ $\approx 6.2 \mathrm{~cm}^{-1}$ ). It is important to note that in the range of $\approx 770-780 \mathrm{~cm}^{-1}$, the $A_{1}^{\mathrm{LO}}\left(\mathrm{Ga}_{1-x} \mathrm{Al} \mathrm{l}_{x} \mathrm{~N}\right) \quad(x \approx 0.30)$ phonon modes are not observed in the nonresonance Raman spectra. This can be due to the very small thickness of the AlGaN layer $(30 \mathrm{~nm})$. It is known that the Raman peak shift of the phonon modes in thin GaN epilayers with respect to its value in bulk GaN $\left(\omega^{\text {bulk }}\left[E_{2}^{\text {high }}(\mathrm{GaN})\right]=569 \mathrm{~cm}^{-1}\right.$ and $\left.\omega^{\text {bulk }}\left[A_{1}^{\mathrm{LO}}(\mathrm{GaN})\right]=735 \mathrm{~cm}^{-1}\right)$ (Ref. 27) can arise due to compressive strains (redshift) and optical phonon confinement effect (blueshift). Unlike the Raman peak shift of the phonon modes from GaN layers due to the phonon confinement effect, the GaN block sizes of the mosaic nitride structure in our case is about $50 \mathrm{~nm}$ (see Table I). This is too large to give rise to the confinement effect and therefore has been ruled out.

The FWHM of the Raman phonon lines reflects the crystal quality. ${ }^{28}$ It should be noted that small values of FWHM of the $E_{2}^{\text {high }}(\mathrm{GaN})$ line $\left(\approx 3.5 \mathrm{~cm}^{-1}\right)$ and the x-ray rocking curve of the 0002 reflection indicate a sufficiently high crystal quality of the GaN layers. Nevertheless, in the Raman spectra, a weak scattering of the $E_{1}(\mathrm{TO})$ phonons $\left(\approx 559 \mathrm{~cm}^{-1}\right)$ was found, which is forbidden by the selection rules, and is caused by the disturbances of the periodical crystal lattice (the presence of extended defects and crystal disordering on a microscale level).

Curve 1 [Figs. 2(a) and 2(b)] corresponds to an axial focal spot position of the laser beam in the sample volume when a very weak Raman phonon line intensity of sapphire $\omega\left[A_{1 g}(s)\right] \approx 418 \mathrm{~cm}^{-1}$ was registered in the Raman spectrum. We used the intensity of this Raman phonon line as a qualitative parameter of the change in the focal spot position along the growth direction of the structure. As follows from the Raman spectra in Fig. 2(a), the phonon lines corresponding to the modes of sapphire with symmetry $E_{g}(s)(\approx 379$, 432, 451, 578, and $751 \mathrm{~cm}^{-1}$ ) (Ref. 29) appear with increasing scanning depth (curves 2-4) and their intensity increases. 

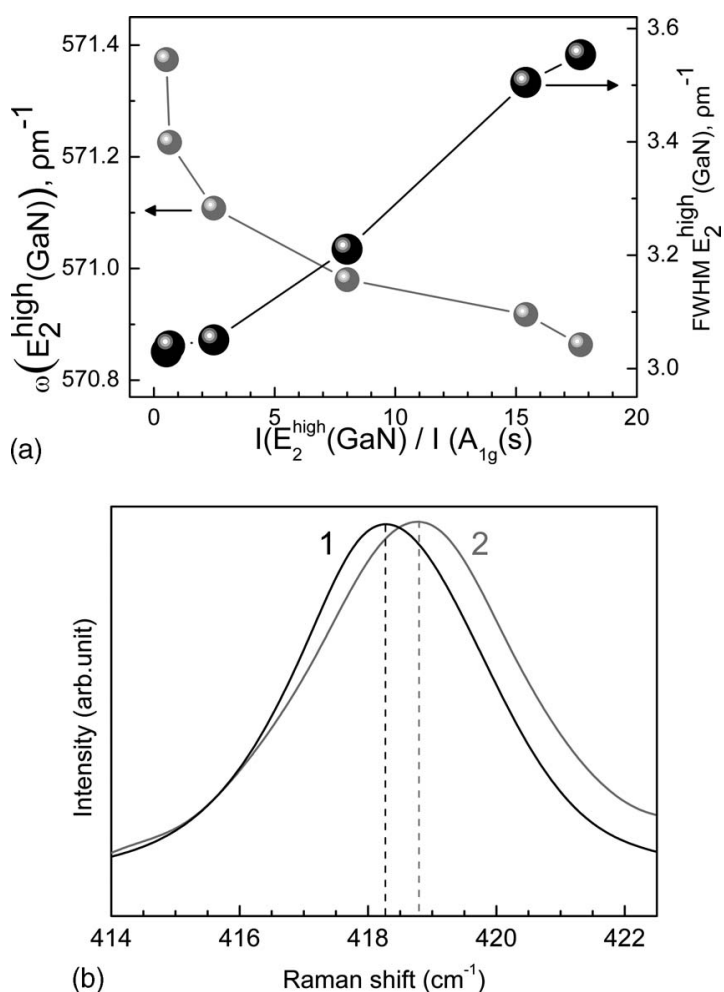

FIG. 3. (a) Frequencies and the FWHM of the $E_{2}^{\text {high }}(\mathrm{GaN})$ phonon mode vs the excitation depth for the $\mathrm{Al}_{0.30} \mathrm{Ga}_{0.70} \mathrm{~N} / \mathrm{GaN}$ structure (Fig. 2), plotted as a function of the ratio of intensities $I\left[E_{2}^{\text {high }}(\mathrm{GaN})\right] / I\left[A_{1 g}(s)\right]$. (b) Raman spectra for the $A_{1 g}(s)$ phonon mode of the sapphire substrate under excitation from the nitride layers side (curve 1) and back side (curve 2) of the investigated structure.

Simultaneously, the intensity of the $E_{2}^{\text {high }}(\mathrm{GaN})$ and the $A_{1}^{\mathrm{LO}}(\mathrm{GaN})$ phonon lines decrease as a function of distance spot position in the depth direction to the GaN/sapphire interface. In addition, curve 5, which is in good agreement with literature data, ${ }^{29}$ corresponds to the Raman spectrum of the sapphire substrate obtained when the back surface of the structure is excited.

The change in frequency and FWHM of the $E_{2}^{\text {high }}(\mathrm{GaN})$ phonon line as a function of the excitation depth is shown in Fig. 3(a). These data demonstrate that the deeper regions closer to the GaN/sapphire interface (i.e., where the ratio of the integrated intensities $I\left[E_{2}^{\text {high }}(\mathrm{GaN})\right] / I\left[A_{1 g}(s)\right]$ has a minimal value) are characterized by the higher frequency and the smaller FWHM of the $E_{2}^{\text {high }}(\mathrm{GaN})$ phonon line [Fig. 2(a), curve 4]. Based on Raman spectra results, we found that the strain (crystal quality) at the GaN/sapphire interface was large (better crystallinity) compared with the surface of the GaN epilayers [Fig. 2(a), curve 1]. The examination of the depth distribution and the relationship between the deformations and crystallinity revealed that the partial compressive deformations relaxed away from the GaN/sapphire interface to the surface, while the crystallinity worsened along the growth direction. We believe that such a behavior was caused by deformation relaxation through defect generation, which results in an increase in FWHM. Thus, the Raman data show the depth distribution of the deformation gradient in the mosaic nitride epilayers at the microscale level.

On the other hand, it is known that the high-frequency
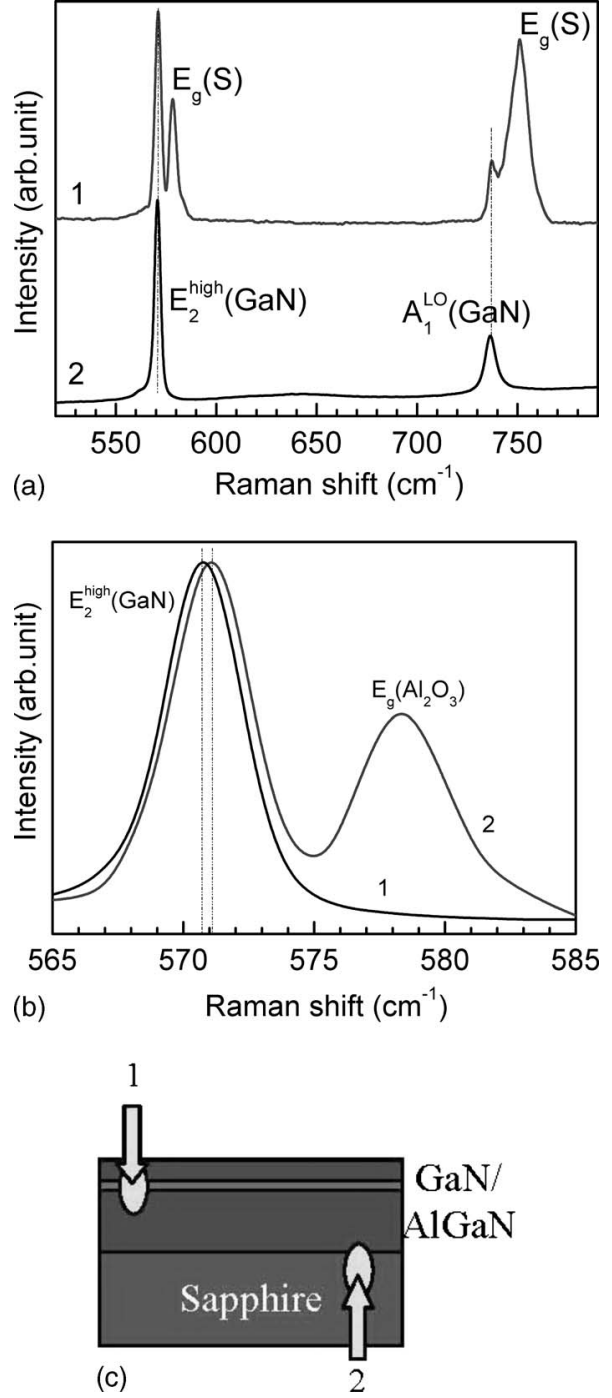

FIG. 4. [(a) and (b)] Raman spectra of the $\mathrm{Al}_{0.30} \mathrm{Ga}_{0.70} \mathrm{~N} / \mathrm{GaN}$ structure grown on a thin sapphire substrate $(450 \mu \mathrm{m})$ measured at excitation from the nitride layers side (curve 1) and back side (curve 2) at depths shown schematically by drawn points 1 and 2 in (c), respectively: $T=300 \mathrm{~K}$ and $\lambda_{\text {exc }}=632.8 \mathrm{~nm}$.

shift of the $A_{1 g}(s)$ sapphire phonon line corresponds to compressive stress along the [0001] growth direction of the structure (or lateral tensile stress). ${ }^{18}$ In our case, the small lowfrequency shift of the $A_{1 g}(s)$ phonon mode was reliably registered in the confocal Raman spectra as we moved away from the GaN/sapphire interface into the depth of the sapphire substrate [Fig. 3(b)]. The shift reflects the elastic compressive deformation relaxation in the sapphire layers near the GaN/sapphire interface. Meanwhile, the maximal highfrequency shift of the $A_{1 g}(s)$ sapphire phonon mode at the GaN/sapphire interface is $\approx 0.5 \mathrm{~cm}^{-1}$, which corresponds to a compressive stress of $\sigma_{z z} \approx 0.5 \mathrm{GPa}^{18}$

The investigation of the micro-Raman spectra of an $\mathrm{AlGaN} / \mathrm{GaN}$ heterostructure grown on a thin sapphire substrate $(450 \mu \mathrm{m})$ showed that the value and character of depth redistribution of deformations are similar in this case to the results obtained above for the structure grown on a thick sapphire substrate $(3 \mathrm{~mm})$. Figure 4(a) shows Raman spectra of the sample grown on a thin sapphire substrate 
using laser excitation from the front surface nitride layers (curve 1) and from the back side of the structure (curve 2), recorded at the depths shown in Fig. 4(c). Upon excitation from the substrate side [curve 2, Fig. 4(a)], the $E_{2}^{\text {high }}(\mathrm{GaN})$ and the $A_{1}^{\mathrm{LO}}(\mathrm{GaN})$ phonon modes and also phonon modes of sapphire can be observed. The analysis of the Raman spectra in the range of $565-585 \mathrm{~cm}^{-1}$ [Fig. 4(b)] shows that the frequency of the $E_{2}^{\text {high }}(\mathrm{GaN})$ phonon mode $\left(\approx 570 \mathrm{~cm}^{-1}\right)$ is slightly higher $\left(\Delta \omega \approx 0.4 \mathrm{~cm}^{-1}\right)$ near the GaN/sapphire interface than that of nitride epilayers near the surface. This fact reflects the relaxation of deformations in GaN epilayers as we move away from the GaN/sapphire interface to the structure's surface.

An analysis of the Raman spectra obtained at an equal probing depth of the investigated structure [equal intensity of $A_{1 g}(s)$ line] demonstrates that the line of the $E_{2}^{\text {high }}(\mathrm{GaN})$ phonon mode has a higher frequency for the sample grown on a thick sapphire substrate (the magnitude of compressive deformations is higher) than that grown on the thin sapphire substrate. It is important to note that the Raman peak shift of the $E_{2}^{\text {high }}(\mathrm{GaN})$ phonon mode and, respectively, the depth distribution of the deformations are practically independent of the substrate thickness. Thus, we can conclude that the deformation depth profile is determined primarily by the elastic deformations depending on the size of the crystallites in the mosaic nitride structures, which are practically the same in the investigated samples (Table I). It should be noted that the depth profile of the deformations in sapphire layers near the GaN/sapphire interface does not depend on the thickness of the substrate. At the same time, the elastic deformations are higher in the nitride layers grown on thick sapphire substrates. This was confirmed by X-ray data.

\section{RESULTS DISCUSSION}

The main source of X-ray reflection broadening in epitaxial systems with dislocation networks is the misorientation of coherent regions. Meanwhile, the contribution introduced by the decreasing sizes of these regions is considerably smaller (for a linear density of the networks below $10^{10} \mathrm{~cm}^{-2}$ ). In addition, a flat configuration of the networks should not cause noticeable changes in coherent sizes perpendicular to the interface direction. However, the observed broadening of the intensity distribution in the direction parallel to the surface (perpendicular to the diffraction vector) definitely demonstrates that the dislocation ensemble contains rectilinear dislocations perpendicular to the interface as well as screw dislocations.

The misorientation of the heterostructure planes is not related to shift deformations [which are difficult to imagine for (0001) orientation of the sample] but are caused by the twisting of the corresponding layers with respect to the substrate or to each other. The twist angles of the planes increase as we move away from the substrate interfacial layer and the substrate sublayer toward the film surface. Therefore, each layer of the dislocation network contributes to twisting in the same direction. This fact is a direct confirmation of the theoretical conception, in which the micromisorientation of the layers and the substrate is determined by the dislocation net- work as well as by the deviation of the crystal surface from the right crystallographic orientation. The height of the steps was calculated using the disorientation angle $\left(0.3^{\circ}\right.$ for thick substrates and $0.12^{\circ}$ for thin substrates) and a distance between the steps (100 and $170 \mathrm{~nm}$, respectively). This was performed in order to confirm the validity of the suggestion that the misorientation influences the parameters of growth steps as resolved by the AFM method. The value of the step height obtained by the calculation is in good agreement with the experimentally obtained value by AFM. The obtained value was about $0.6 \mathrm{~nm}$ for layers grown on TKs and $0.2 \mathrm{~nm}$ for layers grown on TNs.

The difference between the values of the components $\varepsilon_{x x}$ and $\varepsilon_{z z}$ for samples grown on TKs is smaller than that for samples grown on TNs. Therefore, in the case of TKs, we most likely have a nontypical structure containing rectilinear dislocation ensembles with Burger's vector, arranged perpendicularly to the interface but with a more chaotic distribution of dislocations in depth. It should be noted that a large value of $\varepsilon_{z x}$ demonstrates that the system of rectilinear screw dislocations holds in this sample.

What exactly has an influence on the formation of a dislocation structure, on deformations, and on RCS sizes? The misorientation of the surface from its right position is most likely responsible for this finding. However, in this case, the substrate thickness should not play a significant role in the formation of the layer structure. Yet the experimental results demonstrate a different structure formation for the layers grown on TNs and TKs.

Let us consider the influence of thermal expansion coefficients between the GaN film and the substrate on the residual strains in the structures grown on $\mathrm{Al}_{2} \mathrm{O}_{3}$ substrates with different thicknesses (assuming an ideal two-layer structure). If the mismatch strains are fully relaxed at growth temperature, then a difference in interplane lateral distances between the film and the substrate at room temperature in tangential direction can be estimated using

$$
(\Delta a / a)_{\|}^{\prime}=\left(\alpha_{\mathrm{GaN}}-\alpha_{\mathrm{Al}_{2} \mathrm{O}_{3}}\right) \Delta T
$$

where $\Delta T$ is the difference between the growth and measurement temperature, and $\alpha_{\|}^{\mathrm{Al}_{2} \mathrm{O}_{3}}=5.2 \times 10^{-6} \mathrm{~K}^{-1}$ and $\alpha_{\|}^{\mathrm{GaN}}$ $=5.59 \times 10^{-6} \mathrm{~K}^{-1}$ are the coefficients of thermal expansion at room temperature of the substrate and film, respectively

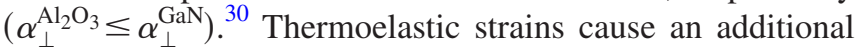
deformation in the direction perpendicular to the $c$-axis of the layer. This differs from the value corresponding to the substrate, but occurs in the opposite direction with respect to the discrepancy jump of the lattice parameters $\left(a_{\mathrm{GaN}}\right.$ $\left.\leq a_{\mathrm{Al}_{2} \mathrm{O}_{3}}\right)$. The influence of the thermogradients is similar to the strains related to lattice discrepancy. However, their values are several orders of magnitude lower than that of the strains.

The mismatch of lattice constants in the growth plane for $\mathrm{GaN}$ and $\mathrm{Al}_{2} \mathrm{O}_{3}$ can be calculated by $(\Delta a / a)_{\|}$ $=\left(a_{\mathrm{GaN}^{-}} a_{\mathrm{Al}_{2} \mathrm{O}_{3}}\right) / a_{\mathrm{Al}_{2} \mathrm{O}_{3}}=-0.33$, where $a_{\mathrm{GaN}}=0.31876 \mathrm{~nm}$ and $a_{\mathrm{Al}_{2} \mathrm{O}_{3}}=0.4758 \mathrm{~nm}$. ${ }^{31}$ Such a mismatch in the lattice constants at a coherent growth should result in a macrobend of the substrate/epilayer system (concavity from the film 
TABLE II. Macrodeformation and curvature radius of the GaN layers.

\begin{tabular}{lcccccccc}
\hline \hline & \multicolumn{2}{c}{$\begin{array}{c}\text { HRXRD } \\
\text { macrodeformation }\end{array}$} & & \multicolumn{2}{c}{$\begin{array}{c}\text { Estimated } \\
\text { macrodeformation }\end{array}$} \\
\cline { 2 - 3 } Samples & $\varepsilon_{x}$ & $\varepsilon_{z}$ & & $\varepsilon_{x}$ & $\varepsilon_{z}$ & $\begin{array}{c}R_{\text {expt }} \\
(\mathrm{m})\end{array}$ & $\begin{array}{c}R_{\text {calc }} \\
(\mathrm{m})\end{array}$ \\
\hline TNs GaN & -0.0137 & 0.00382 & & -0.0722 & $\ldots$ & 9.9 & 0.08 \\
TKs GaN & -0.0026 & 0.00083 & & -0.0174 & $\ldots$ & 69 & 3.5 \\
\hline \hline
\end{tabular}

side). However, in our measurements, the system bend occurs in such a way that the surface of the film is convex.

From the relation between the deformation components, it follows that a dilatation component of microdeformation $\varepsilon_{x x}$ is considerably higher in its $z$ direction value $z-\varepsilon_{z z}$, i.e., local variations in interplane distances for the planes perpendicular to the film surface are considerably higher than those corresponding to parallel planes. The calculated values of the lateral deformations at the interface layer/GaN, taking into account their depth redistribution, are $\varepsilon_{x x}^{\mathrm{Al}_{2} \mathrm{O}_{3}}=4.39 \times 10^{-3}$ and $\varepsilon_{x x}^{\mathrm{GaN}}=-0.1637$ for TNs and $\varepsilon_{x x}^{\mathrm{Al}_{2} \mathrm{O}_{3}}=6.707 \times 10^{-4}$ and $\varepsilon_{x x}^{\mathrm{GaN}}=-0.1674$ for TKs, respectively. ${ }^{32}$ As can be deduced from the data, a deformation jump for the $\mathrm{Al}_{2} \mathrm{O}_{3}$ TKs is one order of magnitude lower than that of TNs. For the GaN film, the jump is practically the same in both cases. In the case of TKs, this should result in the formation of a considerably lower dislocation density at the interface, compensating for the disagreement. This was confirmed experimentally (see Table I). The measured values of curvature radius $R$ for both structures (TNs sample $R=9.9 \mathrm{~m}$; TKs sample $R=69 \mathrm{~m}$ ) differ considerably from the calculated ones (TN sample $R$ $=0.08 \mathrm{~m}$; TKs sample $R=3.5 \mathrm{~m}$ ). The values of the curvature radius and macrodeformations, estimated from these data using the Stoney relation, are presented in Table II.

However, the necessary dislocation density to compensate for the strains exceeds $10^{14}-10^{16} \mathrm{~cm}^{-2}$. This does not agree with the experimental data and can be explained by the following consideration. We suggest that because the film has a columnar structure, the noted effect could be explained by the fact that some of the GaN pillars are arranged in the merger plane at a lateral mismatch of $(\Delta a / a)_{\|}=\left(a_{\mathrm{GaN}}\right.$ $\left.-a_{\mathrm{Al}_{2} \mathrm{O}_{3}}\right) / a_{\mathrm{Al}_{2} \mathrm{O}_{3}}=0.14 \quad\left(a_{\mathrm{GaN}}=0.31876 \mathrm{~nm}\right.$ and $a_{\mathrm{Al}_{2} \mathrm{O}_{3}}$ $=0.2749 \mathrm{~nm}$ ) which, in turn, results in a change in the film deformation sign from tension to compression. The value 0.14 for sapphire was obtained by a $30^{\circ}$ rotation around the GaN [0001] axis with respect to the sapphire unit cell. ${ }^{33}$ Taking this into account, the values of deformations at the film/substrate interface can be calculated as follows: $\varepsilon_{x x}^{\mathrm{Al}_{2} \mathrm{O}_{3}}$ $=-1.2494 \times 10^{-3}$ for TNs and $\varepsilon_{x x}^{\mathrm{GaN}}=0.07223$ for the film, and $\varepsilon_{x x}^{\mathrm{Al}_{2} \mathrm{O}_{3}}=-2.9776 \times 10^{-4}$ for TKs and $\varepsilon_{x x}^{\mathrm{GaN}}=0.017413$ for the film, respectively.

Dislocations with different Burgers vectors may be generated in the layers of the investigated structures. However, as was shown above (Table I), the mismatch dislocation networks parallel to the interface are the dominant dislocation types with Burger's vector parallel to the interface. AlGaN layers have a lower dislocation density related to the smaller mismatch in the lattice parameters with partially relaxed elastic deformations in the heterostructure. Thus, the main difference between the parameters of heterostructure layers grown on thin and thick substrates is caused by different values of deformation jumps at the interface. In the structure grown on TKs, this jump is one order of magnitude lower than the one grown on TNs.

Therefore, when the heterostructures grow at a high temperature, the lattice mismatch strains are practically fully relaxed $\left(\varepsilon_{x x} \approx \varepsilon_{z z}\right.$ ), corresponding to the interface dislocations. Their formation is caused by large lattice mismatch parameters in the $\mathrm{GaN} / \mathrm{AlGaN} / \mathrm{Al}_{2} \mathrm{O}_{3}$ heterostructure. At the same time, the magnitude of the residual elastic deformations in the investigated heterostructures depends on the substrate thickness.

As pointed out previously, the value of the deformations in $\mathrm{GaN}$ epilayers is determined from the Raman peak shift of the nonpolar $E_{2}^{\text {high }}(\mathrm{GaN})$ phonon mode, ${ }^{28}$ which corresponds to lattice vibrations in the plane perpendicular to the [0001] growth direction. ${ }^{34}$ For the case of a strictly biaxial stress, such as in epitaxially grown pseudomorphic layers, the deformation tensor components $\varepsilon_{i j}$ satisfy $\varepsilon_{x y}=\varepsilon_{z y}=\varepsilon_{x z}=0, \varepsilon_{x x}$ $=\varepsilon_{y y}$, where $\varepsilon_{x x}=\left(a-a_{0}\right) / a_{0}$ and $\varepsilon_{z z}=\left(c-c_{0}\right) / c_{0}$. If we apply the approach of pure elastic theory (where Hooke's law is valid), the Raman peak shift of the $E_{2}^{\text {high }}$ phonon mode is related to the strain tensor via $^{35}$

$$
\Delta \omega_{E_{2}}=2 a_{E_{2}} \varepsilon_{x x}+b_{E_{2}} \varepsilon_{z z}=2\left[a_{E_{2}}-b_{E_{2}}\left(C_{13} / C_{33}\right)\right\rfloor \varepsilon_{x x},
$$

where coefficients $a_{E_{2}}$ and $b_{E_{2}}\left(\right.$ in $\left.\mathrm{cm}^{-1}\right)$ are the constants of the phonon deformation potential and $C_{i j}$ are the elastic constants of the material.

For the calculation of the $\varepsilon_{x x}$ deformation using Eq. (2), the following values are used for the constants: $a_{E_{2}}=$ $-850 \pm 25 \mathrm{~cm}^{-1}, \quad b_{E_{2}}=-920 \pm 60 \mathrm{~cm}^{-1},{ }^{14} \quad \omega_{0}\left(E_{2}^{\text {high }}\right)$ $=569 \mathrm{~cm}^{-1}$, and $\left(\varepsilon_{z z} / \varepsilon_{x x}\right)^{B}=-2 C_{13} / C_{33}=-0.6 \pm 0.2 .^{2}$ It was found that with increasing distance from the GaN/sapphire interface, the value $\varepsilon_{x x}$ for TNs $(450 \mu \mathrm{m})$ varies from $\approx 4.18 \times 10^{-3}$ to $\approx 3.3 \times 10^{-3}$, and for TKs $(3 \mathrm{~mm})$ from $\approx 1.7 \times 10^{-3}$ to $\approx 8.7 \times 10^{-4}$. These values of $\varepsilon_{x x}$ are slightly higher than those obtained in X-ray investigations (Table I). The latter is a result of the complexity in deformation analysis for such structures. First, in real mosaic nitride heterostructures, biaxial, uniaxial, and hydrostatic deformations can be formed simultaneously. Each contribution is very difficult to determine separately. At the same time, the phonon deformation-shift coefficient $K\left(\Delta \omega=K \varepsilon_{x x}\right)$ for the $E_{2}^{\text {high }}$ phonon mode depends significantly on the deformation type: ${ }^{36}$ $K_{E_{2}}^{U}=-1.12 \mathrm{~cm}^{-1} / \mathrm{GPa}$ for uniaxial deformations $\left(\varepsilon_{x x}=\varepsilon_{y y}\right.$ $=0), K_{E_{2}}^{B}=-2.43 \mathrm{~cm}^{-1} / \mathrm{GPa}$ for biaxial deformations $\left(\varepsilon_{x x}\right.$ $\left.=\varepsilon_{y y}, \quad \varepsilon_{z z}=0\right)$, and $K_{E_{2}}^{H}=-4.24 \pm 0.03 \mathrm{~cm}^{-1} / \mathrm{GPa}$ for hydrostatic deformations $\left(\varepsilon_{x x}=\varepsilon_{y y}=\varepsilon_{z z}\right)$.

Second, the unusually large fluctuations of elastic deformations, the high concentration of defects and impurities, and the difference between the mosaic structure of the nitride layers (sizes of the blocks and their deviations from vertical growth direction, spatial orientations of the crystallographic planes) cause, in turn, an uncertainty in the anisotropy $\varepsilon_{c} / \varepsilon_{a}-2 C_{13} / C_{33}$ for biaxial deformations. According to Kisielowski et al., ${ }^{2}$ the ratio $\varepsilon_{c} / \varepsilon_{a}$ can be calculated from the recently published data using elastic constants and lattice constants of GaN, which vary greatly. They obtained numeri- 
cal values from -1.5 to 1.5 . Using the experiments on Brillouin scattering in Ref. 37, the values $\varepsilon_{c} / \varepsilon_{a}=0.53 \pm 0.10$ were obtained. On the other hand, the growth parameters (growth rate, temperature, and doping) also significantly influence the structural parameters of the epilayers and, correspondingly, $\varepsilon_{c} / \varepsilon_{a}{ }^{37}$ The situation becomes more complex under the presence of a high density of edge dislocations. This causes a partial relaxation of elastic deformations and a decreasing value of the Poisson relation compared to the structure without dislocations. ${ }^{38}$ On the other hand, the elastic properties of the nanostructures can significantly differ from monocrystals. According to the analysis of Reparaz et $a l .{ }^{39}$ and Pezzoli et al. ${ }^{40}$ on $\mathrm{Ge} / \mathrm{Si}$ thin films and quantum dots (QDs), there is also a great deal of discrepancy between different literature sources regarding the phonon deformation-shift coefficient (up to a factor of 2). This may be due to many reasons such as the geometrical aspect ratio of QDs and cap-layer thickness, spatial nonuniformity of residual deformations throughout the SiGe epilayers, or large uncertainties in the determination of the phonon frequency for the unstrained thin layers in the strained multilayer structures compared to the literature data obtained for bulk materials. In addition, it was shown that under the cover of $\mathrm{Ge}$ QDs by a Si epilayer, the character of the deformations can partially change from biaxial to hydrostatic. ${ }^{39}$ The uncertainties in elastic biaxial macrodeformations result in a significant dispersion of the magnitude of deformation-shifts coefficient $\widetilde{K}_{E_{2}^{\text {high }}}^{B}$ of the $E_{2}^{\text {high }}$ phonon mode from -2.4 to $-10.8 \mathrm{~cm}^{-1} / \mathrm{GPa}^{2,35}$ This led us to the conclusion that there is a difference in the real nitride microstructures between experimental (confocal Raman and X-ray data) and calculated deformations. This difference can concern the uncertainty in their anisotropy or any other deformation components, such as hydrostatic and/or uniaxial deformations, which were not taken into account in our calculations. In reality, the investigation of deformations in mosaic structures (elastic nonuniform media) using Raman scattering is not a simple task because the elastic constants and the phonon deformation potentials must be represented by their average values, reflecting the crystallographic orientation and size of the crystallites. The Voigt-Reuss-Hill averaging technique was therefore used for strain characterization in randomly oriented polycrystalline diamond and silicon films. ${ }^{41}$ The same approximation was used to determine the elastic constants of $\mathrm{GaN}^{2}$

\section{CONCLUSION}

The present results of X-ray and micro-Raman investigations of $\mathrm{Al}_{0.30} \mathrm{Ga}_{0.70} \mathrm{~N} / \mathrm{GaN}$ heterostructures show the following for an increasing sapphire substrate thickness: (i) elastic deformations increase in nitride layers, which is in good agreement with the calculated X-ray data; (ii) the vertical and lateral sizes of the coherent scattering regions (mosaic structure blocks of $\mathrm{GaN}$ layer) decrease from $\approx 65$ to $\approx 32 \mathrm{~nm}$ and increase from $\approx 50$ to $\approx 59 \mathrm{~nm}$, respectively. We found that III-nitride layers have a columnlike structure with small deviations of vertical orientation of the blocks in the growth direction (about $0.38^{\circ}$ ).
We established that the primary structural defects in IIInitride layers are mainly the linear dislocations (screw as well as edge type) perpendicular to the interface. It was shown that confocal micro-Raman spectroscopy is very useful for evaluating the residual deformations and the crystal quality of nitride structures with a microscale spatial resolution. The depth profiles of microdeformations were obtained in the mosaic structure of nitride layers, as well as at the interface region nearly the sapphire substrate. By moving away from the interface substrate/layer, the compressive strains decrease as measured by the Raman peak shift of the $E_{2}^{\text {high }}$ phonon mode. The depth profile of residual deformations in GaN layers depends significantly on the sapphire substrate thickness and its value changes from $\approx 4.18$ $\times 10^{-3}$ to $\approx 3.3 \times 10^{-3}$ and from $\approx 1.7 \times 10^{-3}$ to $\approx 8.7$ $\times 10^{-4}$ for thin and thick substrates, respectively. This corresponds to structure relaxation during the growth process. In addition, the obtained results demonstrate that the significant uncertainties in the values of elastic deformations in the nitrides, as derived from literature data, are caused by the peculiarities of the parameters of the mosaic crystal structure in the nitride epilayers. In summary, the precise determination of the deformation states (particularly, for calculations of the strain parameters and the phonon deformation potentials) in nitride microstructures requires a combination of XRD and micro-Raman spectroscopy methods.

${ }^{1}$ T. Wang, J. Bai, S. Sakai, and J. K. Ho, Appl. Phys. Lett. 78, 2617 (2001). ${ }^{2}$ C. Kisielowski, J. Krüger, S. Ruvimov, T. Suski, J. W. Ager, E. Jones, Z. Liliental-Weber, M. Rubin, E. R. Weber, M. D. Bremser, and R. F. Davis, Phys. Rev. B 54, 17745 (1996).

${ }^{3}$ S. D. Lester, F. A. Ponce, M. G. Craford, and D. A. Steigerwald, Appl. Phys. Lett. 66, 1249 (1995).

${ }^{4}$ Z. Zhong, O. Ambacher, A. Link, V. Holy, J. Stangl, R. T. Lechner, T. Roch, and G. Bauer, Appl. Phys. Lett. 80, 3521 (2002).

${ }^{5}$ L. Kirste, K. M. Pavlov, S. T. Mudie, V. I. Punegov, and N. Herres, J. Appl. Crystallogr. 38, 183 (2005).

${ }^{6}$ C. Q. Chen, Y. Shi, Y. S. Zhang, J. Zhu, and Y. J. Yan, Phys. Rev. Lett. 96, 075505 (2006)

${ }^{7}$ B. Xu, A. J. Lu, B. C. Pan, and Q. X. Yu, Phys. Rev. B 71, 125434 (2005).

${ }^{8}$ A. Filippetti, V. Fiorentini, G. Cappellini, and A. Bosin, Phys. Rev. B 59, 8026 (1999).

${ }^{9}$ N. M. Shmidt, V. V. Emtsev, A. G. Kolmakov, A. D. Kryzhanovsky, W. V. Lundin, D. S. Poloskin, V. V. Ratnikov, A. N. Titkov, A. S. Usikov, and E. E. Zavarin, Nanotechnology 12, 471 (2001).

${ }^{10}$ M. A. Tagliente, L. Tapfer, P. Waltereit, O. Brandt, and K.-H. Ploog, J. Phys. D 36, A192 (2003).

${ }^{11}$ V. V. Ratnikov, R. N. Kyutt, T. V. Shubina, T. Paskova, and B. Monemar, J. Phys. D 34, A30 (2001).

${ }^{12}$ H. F. Poulsen, S. F. Nielsen, E. M. Lauridsen, S. Schmidt, R. M. Suter, U. Lienert, L. Margulies, T. Lorentzen, and D. Juul Jensen, J. Appl. Crystallogr. 34, 751 (2001).

${ }^{13}$ G. E. Ice and B. C. Larson, Adv. Eng. Mater. 2, 643 (2000).

${ }^{14}$ V. Yu. Davydov, N. S. Averkiev, N. S. Goncharuk, D. K. Nelson, I. P. Nikitina, A. S. Polkovnikov, A. N. Smirnov, M. A. Jacobson, and O. K. Semchinova, J. Appl. Phys. 82, 5097 (1997).

${ }^{15}$ T. Prokofyeva, M. Seon, J. Vanbuskirk, M. Holtz, S. A. Nikishin, N. N. Faleev, H. Temkin, and S. Zollner, Phys. Rev. B 63, 125313 (2001).

${ }^{16}$ M. Holtz, T. Prokofyeva, M. Seon, K. Copeland, J. Vanbuskirk, S. Williams, S. A. Nikishin, V. Tretyakov, and H. Temkin, J. Appl. Phys. 89, 7977 (2001)

${ }^{17}$ J. E. N. Jonkman and E. H. K. Stelzer, in Confocal and Two-Photon Microscopy: Foundations, Applications, and Advances, edited by A. Diaspro (Wiley-Liss, New York, 2002), pp. 101-125.

${ }^{18}$ T. Wermelinger, C. Borgia, C. Solenthaler, and R. Spolenak, Acta Mater. 55, 4657 (2007).

${ }^{19}$ V. P. Klad'ko, S. V. Chornen'kii, A. V. Naumov, A. V. Komarov, M. 
Tacano, Yu. N. Svechnikov, S. A. Vitusevich, and A. E. Belyaev, Semiconductors 40, 1060 (2006).

${ }^{20}$ F. Paul, Fewster X-ray Scattering from Semiconductors, 2nd ed. (Imperial College Press, London, 2003).

${ }^{21}$ B. Liu, R. Zhang, Z. L. Xie, H. Lu, Q. J. Liu, Z. Zhang, Y. Li, X. Q. Xiu, P. Chen, P. Han, S. L. Gu, Y. Shi, Y. D. Zheng, and W. J. Schaff, J. Appl. Phys. 103, 023504 (2008).

${ }^{22}$ R. Chierchia, T. B. Wttcher, H. Heinke, S. Einfeldt, S. Figge, and D Hommel, J. Appl. Phys. 93, 8918 (2003).

${ }^{23}$ T. Metzger, R. Hopler, E. Born, O. Ambacher, M. Stutzmann, R. Stommer, M. Schuster, H. Gobel, S. Christiansen, M. Albrecht, and H. P. Strunk, Philos. Mag. A 77, 1013 (1998).

${ }^{24}$ V. Ratnikov, R. Kyutt, T. Shubina, T. Paskova, E. Valcheva, and B. Monemar, J. Appl. Phys. 88, 6252 (2000).

${ }^{25}$ Semiconductors: Data Handbook, 3rd ed., edited by O. Madelung (Springer, Berlin, 2004).

${ }^{26}$ W. Hayers and R. Laudon, Scattering of Light by Crystals (Wiley, New York, 1978).

${ }^{27}$ Y. T. Song, X. L. Chen, W. J. Wang, W. X. Yuan, Y. G. Cao, and X. Wu, J. Cryst. Growth 260, 327 (2004).

${ }^{28}$ H. Grille, Ch. Schnittler, and F. Bechstedt, Phys. Rev. B 61, 6091 (2000).

${ }^{29}$ Z. C. Feng, M. Schurman, and R. A. Stall, J. Vac. Sci. Technol. A 15, 2428 (1997).

${ }^{30}$ Numerical Data and Functional Relationships in Science and Technology, Landolt-Borstein, New Series, Group III, Vol. 17A (Springer, Berlin,
1982).

${ }^{31}$ V. Cimalla, J. Pezoldt, and O. Ambacher, J. Phys. D 40, 6386 (2007).

${ }^{32}$ S. Einfeldt, V. Kirchner, H. Heinke, M. Diebelberg, S. Figge, K. Vogeler, and D. Hommel, J. Appl. Phys. 88, 7029 (2000).

${ }^{33}$ S. C. Jain, M. Willander, J. Narayan, and R. Van Overstraeten, J. Appl. Phys. 87, 965 (2000).

${ }^{34}$ P. Perlin, C. Jauberthie-Carillon, J. P. Itie, A. San Miguel, I. Grzegory, and A. Polian, Phys. Rev. B 45, 83 (1992).

${ }^{35}$ J.-M. Wagner and F. Bechstedt, Phys. Rev. B 66, 115202 (2002).

${ }^{36}$ A. R. Goni, H. Siegle, K. Syassen, C. Thomsen, and J.-M. Wagner, Phys. Rev. B 64, 035205 (2001).

${ }^{37}$ A. Polian, M. Grimsditch, and I. Grzegory, J. Appl. Phys. 79, 3343 (1996).

${ }^{38}$ A. Dadgar, P. Veit, F. Schulze, J. Bläsing, A. Krtschil, H. Witte, A. Diez, T. Hempel, J. Christen, R. Clos, and A. Krost, Thin Solid Films 515, 4356 (2007).

${ }^{39}$ J. S. Reparaz, A. Bernardi, A. R. Goñi, P. D. Lacharmoise, M. I. Alonso, M. Garriga, J. Novák, and I. Vávra, Appl. Phys. Lett. 91, 081914 (2007); J. S. Reparaz, A. Bernardi, A. R. Goni, M. I. Alonso, and M. Garriga, ibid. 92, 081909 (2008); J. S. Reparaz, A. R. Goni, A. Bernardi, M. I. Alonso, and M. Garriga, Phys. Status Solidi B 246, 548 (2009).

${ }^{40}$ F. Pezzoli, E. Bonera, E. Grilli, M. Guzzi, S. Sanguinetti, D. Chrastina, G. Isella, H. von Kanel, E. Wintersberger, J. Stangl, and G. Bauer, J. Appl. Phys. 103, 093521 (2008).

${ }^{41}$ E. Anastassakis and E. Liarokapis, J. Appl. Phys. 62, 3346 (1987) 